Honam Mathematical J. 35 (2013), No. 3, pp. 351-372

http://dx.doi.org/10.5831/HMJ.2013.35.3.351

\title{
REMARKS OF CONGRUENT ARITHMETIC SUMS OF THETA FUNCTIONS DERIVED FROM DIVISOR FUNCTIONS
}

\author{
Aeran Kim, Daeyeoul Kim*, and Nazli Yildiz İkikardes
}

\begin{abstract}
In this paper, we study a distinction the two generating functions : $\varphi^{k}(q)=\sum_{n=0}^{\infty} r_{k}(n) q^{n}$ and $\varphi^{*, k}(q)=\varphi^{k}(q)-\varphi^{k}\left(q^{2}\right)$ $(k=2,4,6,8,10,12,16)$, where $r_{k}(n)$ is the number of representations of $n$ as the sum of $k$ squares. We also obtain some congruences of representation numbers and divisor function.
\end{abstract}

\section{Introduction}

Throughout this paper, $\mathbb{N}$ and $\mathbb{N}_{0}$ will denote the sets of positive integers and $\mathbb{N} \cup\{0\}$, respectively. For $q \in \mathbb{C}$ with $|q|<1$ the $\varphi$ function is defined by

$$
\varphi(q):=\sum_{n=-\infty}^{\infty} q^{n^{2}}
$$

Then we can see that

$$
\varphi^{k}(q)=\sum_{n=0}^{\infty} r_{k}(n) q^{n} .
$$

For $k \in \mathbb{N}$ and $n \in \mathbb{N}_{0}$ we denote the number of representations of $n$ as the sum of $k$ squares by $r_{k}(n)$, that is

$$
r_{k}(n):=\operatorname{card}\left\{\left(x_{1}, \cdots, x_{k}\right) \in \mathbb{Z}^{k} \mid n=x_{1}^{2}+\cdots+x_{k}^{2}\right\} .
$$

The $\varphi$ function is related to the theta function :

$$
\varphi(q)=\theta_{3}(0, q),
$$

Received March 20, 2013. Accepted June 11, 2013.

2010 Mathematics Subject Classification. 11A67.

Key words and phrases. Infinite product, Convolution sums, Congruent sums.

${ }^{*}$ Corresponding author 
where $\theta_{3}(z, q):=1+2 \sum_{n=1}^{\infty} q^{n^{2}} \cos 2 n z$.

Proposition 1.1. ([3, p. 98]) Let $n \in \mathbb{N}$. From Eq. (1) we can obtain

(a)

$$
\varphi^{2}(q)=1+4 \sum_{n=1}^{\infty}\left(\frac{-4}{n}\right) \frac{q^{n}}{1-q^{n}} .
$$

(b)

$$
\varphi^{4}(q)=1+8 \sum_{\substack{n=1 \\ 4 \nmid n}}^{\infty} \frac{n q^{n}}{1-q^{n}}
$$

(c)

$$
\varphi^{6}(q)=1+16 \sum_{n=1}^{\infty} \frac{n^{2} q^{n}}{1+q^{2 n}}-4 \sum_{\substack{n=1 \\ 2 \nmid n}}^{\infty} \frac{(-1)^{(n-1) / 2} n^{2} q^{n}}{1-q^{n}} .
$$

The Legendre-Jacobi-Kronecker symbol for discriminant -4 in Proposition 1.1 is defined for $d \in \mathbb{N}$ by

$$
\left(\frac{-4}{d}\right):=\left\{\begin{array}{lll}
1, & \text { if } d \equiv 1 & (\bmod 4), \\
-1, & \text { if } d \equiv 3 \quad(\bmod 4), \\
0, & \text { if } d \equiv 0 & (\bmod 2) .
\end{array}\right.
$$

We define

$$
\begin{aligned}
& \varphi^{k, *}(q):=\varphi^{k}(q)-\varphi^{k}\left(q^{2}\right):=\sum_{n=1}^{\infty} r_{k}^{*}(n) q^{n} \\
& =\sum_{n=0}^{\infty} r_{k}(n) q^{n}-\sum_{n=0}^{\infty} r_{k}(n) q^{2 n}=\sum_{n=1}^{\infty}\left\{r_{k}(n)-r_{k}\left(\frac{n}{2}\right)\right\} q^{n} .
\end{aligned}
$$

Similarly, we define

$$
\sigma_{s}^{*}(n)=\sum_{\substack{d \mid n \\ n / d \text { odd }}} d^{s}
$$

Then $\sigma_{s}^{*}(n)=\sigma_{s}(n)-\sigma_{s}\left(\frac{n}{2}\right)$.

In Section 2, noting the Proposition 1.1, we solve $\varphi^{k, *}(q)$ for $k=$ $2,4,6$. And we obtain the property $r_{4}^{*}(n) \equiv 8 \sigma_{1}^{*}(n)(\bmod 32)$. In Section 3, we calculate $\varphi^{k, *}(q)$ for $k=8,10,12$. In Section 4, using the convolution sum for $r_{k}(n)$, we obtain $r_{16}(n)$. Through $r_{16}(n)$ we deduce some congruence relation by modulo 32 and 64 (see Theorem 
4.7). And we also get the relation $b(n)=-8 b\left(\frac{n}{2}\right)$ with even $n$ for $\sum_{n=1}^{\infty} b(n) q^{n}:=q \prod_{n=1}^{\infty}\left(1-q^{n}\right)^{8}\left(1-q^{2 n}\right)^{8}$. Using the formula of $\varphi^{16, *}(q)$, we obtain $r_{16}(n) \equiv 32 \sigma_{7}(n)(\bmod 512)$ for odd $n$ (Theorem 4.9). Finally in Appendix we list some values for $b(n)$.

\section{The property of $\varphi^{k, *}(q)$}

Lemma 2.1. Let $n \in \mathbb{N}$. Then we have

$$
\varphi^{2, *}(q)=4 \sum_{\substack{d \mid n \\ d \text { odd }}}\left(\frac{-4}{d}\right) q^{n}=4 \sum_{n=1}^{\infty}\left(\frac{-4}{n}\right) \frac{q^{n}}{1-q^{2 n}} .
$$

Proof. As $r_{2}(n)=4 \sum_{d \mid n}\left(\frac{-4}{d}\right)$, we obtain

$$
r_{2}(2 n)-r_{2}(n)=4\left\{\sum_{d \mid 2 n}\left(\frac{-4}{d}\right)-\sum_{d \mid n}\left(\frac{-4}{d}\right)\right\} .
$$

Here, by (2), we observe that

$$
\sum_{d \mid 2 n}\left(\frac{-4}{d}\right)=\sum_{\substack{d \mid 2 n \\ d \text { odd }}}\left(\frac{-4}{d}\right)=\sum_{\substack{d \mid n \\ d \text { odd }}}\left(\frac{-4}{d}\right)=\sum_{d \mid n}\left(\frac{-4}{d}\right) .
$$

Thus $r_{2}(2 n)-r_{2}(n)=0$, which leads $r_{2}^{*}(2 n)=0$. From this fact and (3) we can see that

$$
\begin{aligned}
\varphi^{2, *}(q) & =\sum_{n=1}^{\infty} r_{2}^{*}(n) q^{n}=\sum_{\substack{n=1 \\
n \text { odd }}}^{\infty} r_{2}^{*}(n) q^{n}=\sum_{\substack{n=1 \\
n \text { odd }}}^{\infty}\left\{r_{2}(n)-r_{2}\left(\frac{n}{2}\right)\right\} q^{n} \\
& =\sum_{\substack{n=1 \\
n \text { odd }}}^{\infty} r_{2}(n) q^{n}=4 \sum_{\substack{d \mid n \\
n \text { odd }}}\left(\frac{-4}{d}\right) q^{n} .
\end{aligned}
$$

By Proposition 1.1 (a) and (3), we get

$$
\begin{aligned}
\varphi^{2, *}(q) & =\varphi^{2}(q)-\varphi^{2}\left(q^{2}\right) \\
& =1+4 \sum_{n=1}^{\infty}\left(\frac{-4}{n}\right) \frac{q^{n}}{1-q^{n}}-\left\{1+4 \sum_{n=1}^{\infty}\left(\frac{-4}{n}\right) \frac{q^{2 n}}{1-q^{2 n}}\right\} \\
& =4 \sum_{n=1}^{\infty}\left(\frac{-4}{n}\right)\left\{\frac{q^{n}}{1-q^{n}}-\frac{q^{2 n}}{1-q^{2 n}}\right\}
\end{aligned}
$$




$$
\begin{aligned}
& =4 \sum_{n=1}^{\infty}\left(\frac{-4}{n}\right) \cdot \frac{q^{n}}{1-q^{2 n}}\left(1+q^{n}-q^{n}\right) \\
& =4 \sum_{n=1}^{\infty}\left(\frac{-4}{n}\right) \frac{q^{n}}{1-q^{2 n}} .
\end{aligned}
$$

Thus the proof is complete.

Lemma 2.2. Let $n \in \mathbb{N}$. Then we have

$$
\varphi^{4, *}(q)=\sum_{n=1}^{\infty}\left\{8 \sigma_{1}^{*}(n)-32 \sigma_{1}^{*}\left(\frac{n}{4}\right)\right\} q^{n}=8 \sum_{\substack{n=1 \\ 4 \nmid n}}^{\infty} n \frac{q^{n}}{1-q^{2 n}} .
$$

Proof. It is clear by Proposition 1.1 (b).

Lemma 2.3. Let $n \in \mathbb{N}$. Then we have

$$
\begin{aligned}
\varphi^{6, *}(q)= & \sum_{n=1}^{\infty}\left\{16 \sum_{\substack{d \mid n \\
n \text { odd }}}\left(\frac{-4}{n / d}\right) d^{2} q^{n}-4 \sum_{n \text { odd }} d \mid n d\right. \\
& \left.+48 \sum_{\substack{d \mid \frac{n}{2} \\
n \text { even }}}\left(\frac{-4}{\frac{n}{2} / d}\right) d^{2} q^{n}\right\} \\
= & 16 \sum_{n=1}^{\infty} n^{2} \frac{q^{n}\left(1-q^{n}\right)\left(1-q^{3 n}\right)}{\left(1+q^{2 n}\right)\left(1+q^{4 n}\right)}-4 \sum_{\substack{n=1 \\
2 \nmid n}}^{\infty}(-1)^{(n-1) / 2} n^{2} \frac{q^{n}}{1-q^{2 n}} .
\end{aligned}
$$

Proof. There are given $r_{6}(n)=16 \sum_{d \mid n}\left(\frac{-4}{n / d}\right) d^{2}-4 \sum_{d \mid n}\left(\frac{-4}{d}\right) d^{2}$ in [3, Theorem 9.6]. So

$$
\begin{aligned}
& r_{6}(2 n)-r_{6}(n) \\
& =16 \sum_{d \mid 2 n}\left(\frac{-4}{2 n / d}\right) d^{2}-4 \sum_{d \mid 2 n}\left(\frac{-4}{d}\right) d^{2}-\left\{16 \sum_{d \mid n}\left(\frac{-4}{n / d}\right) d^{2}-4 \sum_{d \mid n}\left(\frac{-4}{d}\right) d^{2}\right\} \\
& =16\left\{\sum_{d \mid 2 n}\left(\frac{-4}{2 n / d}\right) d^{2}-\sum_{d \mid n}\left(\frac{-4}{n / d}\right) d^{2}\right\}-4\left\{\sum_{d \mid 2 n}\left(\frac{-4}{d}\right) d^{2}-\sum_{d \mid n}\left(\frac{-4}{d}\right) d^{2}\right\} .
\end{aligned}
$$


To simplify the first term in (4), we use the property of (2) and so we have

$$
\begin{aligned}
\sum_{d \mid 2 n}\left(\frac{-4}{2 n / d}\right) d^{2} & =\sum_{\frac{2 n}{d} \text { odd }}\left(\frac{-4}{2 n / d}\right) d^{2}=\sum_{\substack{e \mid 2 n \\
e \text { odd }}}\left(\frac{-4}{e}\right)\left(\frac{2 n}{e}\right)^{2} \\
& =4 \sum_{\substack{e \mid n \\
e \text { odd }}}\left(\frac{-4}{e}\right)\left(\frac{n}{e}\right)^{2},
\end{aligned}
$$

where we put $e:=\frac{2 n}{d}$. By letting $l=\frac{n}{e},(5)$ becomes

(6)

$$
\begin{aligned}
4 \sum_{\substack{e \mid n \\
e \text { odd }}}\left(\frac{-4}{e}\right)\left(\frac{n}{e}\right)^{2} & =4 \sum_{\substack{l \mid n \\
\frac{n}{l} \text { odd }}}\left(\frac{-4}{n / l}\right) l^{2}=4 \sum_{l \mid n}\left(\frac{-4}{n / l}\right) l^{2} \\
& =4 \sum_{d \mid n}\left(\frac{-4}{n / d}\right) d^{2},
\end{aligned}
$$

in the last part we replace the index $l$ with $d$. From (5) and (6), we derive that

$$
\sum_{d \mid 2 n}\left(\frac{-4}{2 n / d}\right) d^{2}-\sum_{d \mid n}\left(\frac{-4}{n / d}\right) d^{2}=3 \sum_{d \mid n}\left(\frac{-4}{n / d}\right) d^{2}
$$

and

(8) $\sum_{\substack{d \mid n \\ n \text { even }}}\left(\frac{-4}{n / d}\right) d^{2}-\sum_{\substack{d \mid \frac{n}{2} \\ n \text { even }}}\left(\frac{-4}{\frac{n}{2} / d}\right) d^{2}=3 \sum_{\substack{d \mid \frac{n}{2} \\ n \text { even }}}\left(\frac{-4}{\frac{n}{2} / d}\right) d^{2}$.

And for the second term in (4) we have

$$
\sum_{d \mid 2 n}\left(\frac{-4}{d}\right) d^{2}=\sum_{\substack{d \mid 2 n \\ d \text { odd }}}\left(\frac{-4}{d}\right) d^{2}=\sum_{\substack{d \mid n \\ d \text { odd }}}\left(\frac{-4}{d}\right) d^{2}=\sum_{d \mid n}\left(\frac{-4}{d}\right) d^{2}
$$

and

$$
\sum_{d \mid 2 n}\left(\frac{-4}{d}\right) d^{2}-\sum_{d \mid n}\left(\frac{-4}{d}\right) d^{2}=0
$$

It implies that

$$
\sum_{\substack{d \mid n \\ n \text { even }}}\left(\frac{-4}{d}\right) d^{2}-\sum_{\substack{d \mid \frac{n}{2} \\ n \text { even }}}\left(\frac{-4}{d}\right) d^{2}=0
$$


From (8) and (9) we deduce that

$$
\begin{aligned}
\varphi^{6, *}(q)= & \sum_{n=1}^{\infty}\left\{r_{6}(n)-r_{6}\left(\frac{n}{2}\right)\right\} q^{n} \\
= & \sum_{n=1}^{\infty}\left[16 \sum_{d \mid n}\left(\frac{-4}{n / d}\right) d^{2}-4 \sum_{d \mid n}\left(\frac{-4}{d}\right) d^{2}\right. \\
& \left.-\left\{16 \sum_{d \mid \frac{n}{2}}\left(\frac{-4}{\frac{n}{2} / d}\right) d^{2}-4 \sum_{d \mid \frac{n}{2}}\left(\frac{-4}{d}\right) d^{2}\right\}\right] q^{n} \\
=\sum_{n=1}^{\infty}\left[16\left\{\sum_{d \mid n}\left(\frac{-4}{n / d}\right) d^{2}-\sum_{d \mid \frac{n}{2}}\left(\frac{-4}{\frac{n}{2} / d}\right) d^{2}\right\}\right. & \left.-4\left\{\sum_{d \mid n}\left(\frac{-4}{d}\right) d^{2}-\sum_{d \mid \frac{n}{2}}\left(\frac{-4}{d}\right) d^{2}\right\}\right] q^{n} \\
= & \sum_{n=1}^{\infty}\left\{16 \sum_{\begin{array}{c}
d \mid n \\
n \text { odd }
\end{array}}\left(\frac{-4}{n / d}\right) d^{2} q^{n}+48 \sum_{n \mid \frac{n}{2}}\left(\frac{-4}{\frac{n}{2} / d}\right) d^{2} q^{n}\right. \\
& \left.-4 \sum_{\substack{d \mid n \\
n \text { odd }}}\left(\frac{-4}{d}\right) d^{2} q^{n}\right\} .
\end{aligned}
$$

By Proposition 1.1 (c), we obtain

$$
\begin{aligned}
& \varphi^{6, *}(q)=\varphi^{6}(q)-\varphi^{6}\left(q^{2}\right) \\
& =\left\{1+16 \sum_{n=1}^{\infty} \frac{n^{2} q^{n}}{1+q^{2 n}}-4 \sum_{\substack{n=1 \\
2 \nmid n}}^{\infty} \frac{(-1)^{(n-1) / 2} n^{2} q^{n}}{1-q^{n}}\right\} \\
& -\left\{1+16 \sum_{n=1}^{\infty} \frac{n^{2} q^{2 n}}{1+q^{4 n}}-4 \sum_{\substack{n=1 \\
2 \nmid n}}^{\infty} \frac{(-1)^{(n-1) / 2} n^{2} q^{2 n}}{1-q^{2 n}}\right\} \\
& =16 \sum_{n=1}^{\infty} n^{2} \frac{q^{n}}{\left(1+q^{2 n}\right)\left(1+q^{4 n}\right)}\left\{1+q^{4 n}-q^{n}\left(1+q^{2 n}\right)\right\}
\end{aligned}
$$




$$
\begin{aligned}
& -4 \sum_{\substack{n=1 \\
2 \nmid n}}^{\infty}(-1)^{(n-1) / 2} n^{2} \cdot \frac{q^{n}}{1-q^{2 n}}\left(1+q^{n}-q^{n}\right) \\
= & 16 \sum_{n=1}^{\infty} n^{2} \frac{q^{n}\left(1-q^{n}\right)\left(1-q^{3 n}\right)}{\left(1+q^{2 n}\right)\left(1+q^{4 n}\right)}-4 \sum_{\substack{n=1 \\
2 \nmid n}}^{\infty}(-1)^{(n-1) / 2} n^{2} \frac{q^{n}}{1-q^{2 n}} .
\end{aligned}
$$

Corollary 2.4. Let $n \in \mathbb{N}$. Then we have $r_{4}^{*}(n) \equiv 8 \sigma_{1}^{*}(n)(\bmod 32)$.

Proof. It is obvious by Lemma 2.2 .

\section{Property of $\varphi$ function}

From (1) we know that $\varphi^{8}(q)=1+\sum_{n=1}^{\infty} r_{8}(n) q^{n}$. By [3, Theorem 19.1] we deduce that

$$
\begin{aligned}
\varphi^{8}(q) & =1+\sum_{n=1}^{\infty}\left\{16(-1)^{n} \sum_{\substack{d \in \mathbb{N} \\
d \mid n}}(-1)^{d} d^{3}\right\} q^{n} \\
& =1+16 \sum_{\substack{n=1 \\
2 \nmid n}}^{\infty} n^{3} \sum_{k=1}^{\infty}(-1)^{k} q^{n k}+16 \sum_{\substack{n=1 \\
2 \mid n}}^{\infty} n^{3} \sum_{k=1}^{\infty} q^{n k} .
\end{aligned}
$$

Lemma 3.1. Let $n \in \mathbb{N}$. Then we have

$$
\begin{aligned}
\varphi^{8, *}(q) & =\sum_{n=1}^{\infty}\left\{16 \sigma_{3}^{*}(n)-32 \sigma_{3}^{*}\left(\frac{n}{2}\right)+256 \sigma_{3}^{*}\left(\frac{n}{4}\right)\right\} q^{n} \\
& =16\left[\sum_{\substack{n=1 \\
2 \nmid n}}^{\infty} n^{3} \frac{q^{n}\left(1-q^{n}\right)}{\left(1+q^{n}\right)\left(1+q^{2 n}\right)}+\sum_{\substack{n=1 \\
2 \mid n}}^{\infty} n^{3} \frac{q^{n}}{1-q^{2 n}}\right] .
\end{aligned}
$$

Proof. Since $r_{8}(n)=16 \sigma_{3}(n)-32 \sigma_{3}\left(\frac{n}{2}\right)+256 \sigma_{3}\left(\frac{n}{4}\right)$ in $[3,(19.10)]$, we have

$$
\begin{aligned}
\varphi^{8, *}(q) & =\sum_{n=1}^{\infty}\left\{r_{8}(n)-r_{8}\left(\frac{n}{2}\right)\right\} q^{n} \\
& =\sum_{n=1}^{\infty}\left[\left\{16 \sigma_{3}(n)-32 \sigma_{3}\left(\frac{n}{2}\right)+256 \sigma_{3}\left(\frac{n}{4}\right)\right\}\right.
\end{aligned}
$$




$$
\begin{array}{r}
\left.-\left\{16 \sigma_{3}\left(\frac{n}{2}\right)-32 \sigma_{3}\left(\frac{n}{4}\right)+256 \sigma_{3}\left(\frac{n}{8}\right)\right\}\right] q^{n} \\
=\sum_{n=1}^{\infty}\left\{16 \sigma_{3}^{*}(n)-32 \sigma_{3}^{*}\left(\frac{n}{2}\right)+256 \sigma_{3}^{*}\left(\frac{n}{4}\right)\right\} q^{n} .
\end{array}
$$

And using (10) we obtain

$$
\begin{aligned}
\varphi^{8, *}(q)=1 & +16\left\{\sum_{\substack{n=1 \\
2 \nmid n}}^{\infty} \frac{n^{3} q^{n}}{1+q^{n}}+\sum_{\substack{n=1 \\
2 \mid n}}^{\infty} \frac{n^{3} q^{n}}{1-q^{n}}\right\} \\
& -\left[1+16\left\{\sum_{\substack{n=1 \\
2 \nmid n}}^{\infty} \frac{n^{3} q^{2 n}}{1+q^{2 n}}+\sum_{\substack{n=1 \\
2 \mid n}}^{\infty} \frac{n^{3} q^{2 n}}{1-q^{2 n}}\right\}\right] \\
=16 & {\left[\sum_{\substack{n=1 \\
2 \nmid n}}^{\infty} n^{3} \frac{q^{n}\left(1-q^{n}\right)}{\left(1+q^{n}\right)\left(1+q^{2 n}\right)}+\sum_{\substack{n=1 \\
2 \mid n}}^{\infty} n^{3} \frac{q^{n}}{1-q^{2 n}}\right] . }
\end{aligned}
$$

In $[3$, p. 260], we can know that

$$
r_{10}(n)=\frac{4}{5} \sum_{d \mid n}\left(\frac{-4}{d}\right) d^{4}+\frac{64}{5} \sum_{d \mid n}\left(\frac{-4}{n / d}\right) d^{4}+\frac{32}{5} w(n),
$$

where $\sum_{n=1}^{\infty} w(n) q^{n}:=q \prod_{n=1}^{\infty}\left(1-q^{n}\right)^{4}\left(1-q^{2 n}\right)^{2}\left(1-q^{4 n}\right)^{4}$. So we obtain that

$$
\varphi^{10}(q)=1+\frac{4}{5} \sum_{\substack{n=1 \\ d \mid n}}^{\infty}\left(\frac{-4}{d}\right) d^{4} q^{n}+\frac{64}{5} \sum_{\substack{n=1 \\ d \mid n}}^{\infty}\left(\frac{-4}{n / d}\right) d^{4} q^{n}+\sum_{n=1}^{\infty} \frac{32}{5} w(n) q^{n} .
$$

Then, by (2), the second term in the right hand side of Eq. (11) can be written as 


$$
\begin{aligned}
\sum_{\substack{n=1 \\
d \mid n}}^{\infty}\left(\frac{-4}{d}\right) d^{4} q^{n}= & \left(\frac{-4}{1}\right) 1^{4} q+\left\{\left(\frac{-4}{1}\right) 1^{4}+\left(\frac{-4}{2}\right) 2^{4}\right\} q^{2} \\
& +\left\{\left(\frac{-4}{1}\right) 1^{4}+\left(\frac{-4}{3}\right) 3^{4}\right\} q^{3} \\
& +\left\{\left(\frac{-4}{1}\right) 1^{4}+\left(\frac{-4}{2}\right) 2^{4}+\left(\frac{-4}{4}\right) 4^{4}\right\} q^{4} \\
& +\cdots \cdots \cdots \\
= & \sum_{\substack{n=1 \\
2 \nmid n}}^{\infty} \frac{(-1)^{\frac{n-1}{2}} n^{4} q^{n}}{1-q^{n}}
\end{aligned}
$$

We note that $\frac{q^{n}}{1-q^{n}}=q^{n}+q^{2 n}+q^{3 n}+\cdots$. Similarly, the third term in (11) becomes

$$
\begin{aligned}
\sum_{\substack{n=1 \\
d \mid n}}^{\infty}\left(\frac{-4}{n / d}\right) d^{4} q^{n}= & 1^{4}\left(q-q^{3}+q^{5}+\cdots\right)+2^{4}\left(q^{2}-q^{6}+q^{10}+\cdots\right) \\
& \quad+3^{4}\left(q^{3}-q^{9}+q^{15}+\cdots\right)+4^{4}\left(q^{4}-q^{12}+q^{20}+\cdots\right) \\
& \quad+\cdots \cdots \cdots \\
= & \sum_{n=1}^{\infty} \frac{n^{4} q^{n}}{1+q^{2 n}} .
\end{aligned}
$$

Therefore, we obtain

$$
\varphi^{10}(q)=1+\frac{4}{5} \sum_{\substack{n=1 \\ 2 \nmid n}}^{\infty} \frac{(-1)^{\frac{n-1}{2}} n^{4} q^{n}}{1-q^{n}}+\frac{64}{5} \sum_{n=1}^{\infty} \frac{n^{4} q^{n}}{1+q^{2 n}}+\frac{32}{5} \sum_{n=1}^{\infty} w(n) q^{n} .
$$

Lemma 3.2. Let $n \in \mathbb{N}$. Then we obtain 


$$
\begin{aligned}
\varphi^{10, *}(q)= & \sum_{n=1}^{\infty}\left\{\frac{4}{5} \sum_{\substack{d \mid n \\
n \text { odd }}}\left(\frac{-4}{d}\right) d^{4} q^{n}+\frac{64}{5} \sum_{\substack{d \mid n \\
\text { odd }}}^{d \mid n}\left(\frac{-4}{n / d}\right) d^{4} q^{n}\right. \\
& \left.+192 \sum_{\substack{d \mid \frac{n}{2} \\
n \text { even }}}\left(\frac{-4}{\frac{n}{2} / d}\right) d^{4} q^{n}+\frac{24}{5} w^{*}(n) q^{n}\right\} \\
= & \frac{4}{5} \sum_{\substack{n=1 \\
2 \nmid n}}^{\infty}(-1)^{(n-1) / 2} n^{4} \frac{q^{n}}{1-q^{2 n}}+\frac{64}{5} \sum_{n=1}^{\infty} n^{4} \frac{q^{n}\left(1-q^{n}\right)\left(1-q^{3 n}\right)}{\left(1+q^{2 n}\right)\left(1+q^{4 n}\right)} \\
& +\frac{32}{5} \sum_{n=1}^{\infty} w^{*}(n) q^{n},
\end{aligned}
$$

where

$$
\begin{aligned}
\sum_{n=1}^{\infty} w^{*}(n) q^{n}: & =\sum_{n=1}^{\infty}\left\{w(n)-w\left(\frac{n}{2}\right)\right\} q^{n} \\
= & q \prod_{n=1}^{\infty}\left(1-q^{n}\right)^{4}\left(1-q^{2 n}\right)^{2}\left(1-q^{4 n}\right)^{4} \\
& \quad-q^{2} \prod_{n=1}^{\infty}\left(1-q^{2 n}\right)^{4}\left(1-q^{4 n}\right)^{2}\left(1-q^{8 n}\right)^{4}
\end{aligned}
$$

Proof. Since $r_{10}(n)=\frac{4}{5} \sum_{d \mid n}\left(\frac{-4}{d}\right) d^{4}+\frac{64}{5} \sum_{d \mid n}\left(\frac{-4}{n / d}\right) d^{4}+\frac{32}{5} w(n)$, so

$$
\begin{aligned}
& r_{10}(2 n)-r_{10}(n) \\
& =\frac{4}{5} \sum_{d \mid 2 n}\left(\frac{-4}{d}\right) d^{4}+\frac{64}{5} \sum_{d \mid 2 n}\left(\frac{-4}{2 n / d}\right) d^{4}+\frac{32}{5} w(2 n) \\
& \quad-\left\{\frac{4}{5} \sum_{d \mid n}\left(\frac{-4}{d}\right) d^{4}+\frac{64}{5} \sum_{d \mid n}\left(\frac{-4}{n / d}\right) d^{4}+\frac{32}{5} w(n)\right\} \\
& =\frac{4}{5}\left\{\sum_{d \mid 2 n}\left(\frac{-4}{d}\right) d^{4}-\sum_{d \mid n}\left(\frac{-4}{d}\right) d^{4}\right\}+\frac{64}{5}\left\{\sum_{d \mid 2 n}\left(\frac{-4}{2 n / d}\right) d^{4}\right. \\
& \left.\quad-\sum_{d \mid n}\left(\frac{-4}{n / d}\right) d^{4}\right\}+\frac{32}{5}\{w(2 n)-w(n)\} .
\end{aligned}
$$

To simplify the first term in (13), we use the property of (2) and so we have 


$$
\begin{aligned}
\sum_{\substack{d \in \mathbb{N} \\
d \mid 2 n}}\left(\frac{-4}{2 n / d}\right) d^{4} & =\sum_{\substack{d \mid 2 n \\
\frac{2 n}{d} \text { odd }}}\left(\frac{-4}{2 n / d}\right) d^{4}=\sum_{\substack{e \mid 2 n \\
e \text { odd }}}\left(\frac{-4}{e}\right)\left(\frac{2 n}{e}\right)^{4} \\
& =16 \sum_{\substack{e \mid n \\
e \text { odd }}}\left(\frac{-4}{e}\right)\left(\frac{n}{e}\right)^{4},
\end{aligned}
$$

where we put $e:=\frac{2 n}{d}$. By letting $l=\frac{n}{e},(14)$ becomes

$$
\begin{aligned}
16 \sum_{\substack{e \mid n \\
e \text { odd }}}\left(\frac{-4}{e}\right)\left(\frac{n}{e}\right)^{4} & =16 \sum_{\substack{l \mid n \\
\frac{n}{l} \text { odd }}}\left(\frac{-4}{n / l}\right) l^{4}=16 \sum_{l \mid n}\left(\frac{-4}{n / l}\right) l^{4} \\
& =16 \sum_{d \mid n}\left(\frac{-4}{n / d}\right) d^{4}
\end{aligned}
$$

in the last part we replace the index $l$ with $d$. (14) and (15) show that

$$
\sum_{d \mid 2 n}\left(\frac{-4}{2 n / d}\right) d^{4}-\sum_{d \mid n}\left(\frac{-4}{n / d}\right) d^{4}=15 \sum_{d \mid n}\left(\frac{-4}{n / d}\right) d^{4}
$$

(16) implies that

$$
\sum_{\substack{d \mid n \\ n \text { even }}}\left(\frac{-4}{n / d}\right) d^{4}-\sum_{\substack{d \mid \frac{n}{2} \\ n \text { even }}}\left(\frac{-4}{\frac{n}{2} / d}\right) d^{4}=15 \sum_{\substack{d \mid \frac{n}{2} \\ n \text { even }}}\left(\frac{-4}{\frac{n}{2} / d}\right) d^{4} .
$$

And for the second term in (13) we have

$$
\sum_{d \mid 2 n}\left(\frac{-4}{d}\right) d^{4}=\sum_{\substack{d \mid 2 n \\ d \text { odd }}}\left(\frac{-4}{d}\right) d^{4}=\sum_{\substack{d \mid n \\ d \text { odd }}}\left(\frac{-4}{d}\right) d^{4}=\sum_{d \mid n}\left(\frac{-4}{d}\right) d^{4} .
$$

So

$$
\sum_{d \mid 2 n}\left(\frac{-4}{d}\right) d^{4}-\sum_{d \mid n}\left(\frac{-4}{d}\right) d^{4}=0 .
$$

It implies that

$$
\sum_{\substack{d \mid n \\ n \text { even }}}\left(\frac{-4}{d}\right) d^{4}-\sum_{\substack{d \mid \frac{n}{2} \\ n \text { even }}}\left(\frac{-4}{d}\right) d^{4}=0
$$


From (17) and (18) we deduce that

$$
\begin{aligned}
& \varphi^{10, *}(q)=\sum_{n=1}^{\infty}\left\{r_{10}(n)-r_{10}\left(\frac{n}{2}\right)\right\} q^{n} \\
&=\sum_{n=1}^{\infty} {\left[\frac{4}{5} \sum_{d \mid n}\left(\frac{-4}{d}\right) d^{4}+\frac{64}{5} \sum_{d \mid n}\left(\frac{-4}{n / d}\right) d^{4}+\frac{32}{5} w(n)\right.} \\
&\left.-\left\{\frac{4}{5} \sum_{d \mid \frac{n}{2}}\left(\frac{-4}{d}\right) d^{4}+\frac{64}{5} \sum_{d \mid \frac{n}{2}}\left(\frac{-4}{\frac{n}{2} / d}\right) d^{4}+\frac{32}{5} w\left(\frac{n}{2}\right)\right\}\right] q^{n} \\
&=\sum_{n=1}^{\infty}\left\{\frac{4}{5} \sum_{\substack{d \mid n \\
n \text { odd }}}\left(\frac{-4}{d}\right) d^{4} q^{n}+\frac{64}{5} \sum_{n \text { odd }}\left(\frac{-4}{n / d}\right) d^{4} q^{n}\right. \\
&\left.+192 \sum_{\substack{d \mid \frac{n}{2} \\
n \text { even }}}\left(\frac{-4}{\frac{n}{2} / d}\right) d^{4} q^{n}+\frac{24}{5} w^{*}(n) q^{n}\right\} .
\end{aligned}
$$

Here, considering the definition of $\sum_{n=1}^{\infty} w(n) q^{n}:=q \prod_{n=1}^{\infty}\left(1-q^{n}\right)^{4}(1-$ $\left.q^{2 n}\right)^{2}\left(1-q^{4 n}\right)^{4}$ we observe that

$$
\begin{aligned}
\sum_{n=1}^{\infty}\left\{w(n)-w\left(\frac{n}{2}\right)\right\} q^{n}=\sum_{n=1}^{\infty}\left\{w(n) q^{n}-w(n) q^{2 n}\right\} \\
=q \prod_{n=1}^{\infty}\left(1-q^{n}\right)^{4}\left(1-q^{2 n}\right)^{2}\left(1-q^{4 n}\right)^{4} \\
\quad-q^{2} \prod_{n=1}^{\infty}\left(1-q^{2 n}\right)^{4}\left(1-q^{4 n}\right)^{2}\left(1-q^{8 n}\right)^{4} .
\end{aligned}
$$

By (12), we get

$$
\begin{aligned}
\varphi^{10, *}(q) & =\varphi^{10}(q)-\varphi^{10}\left(q^{2}\right) \\
= & \left\{+\frac{4}{5} \sum_{\substack{n=1 \\
2 \nmid n}}^{\infty} \frac{(-1)^{\frac{n-1}{2}} n^{4} q^{n}}{1-q^{n}}+\frac{64}{5} \sum_{n=1}^{\infty} \frac{n^{4} q^{n}}{1+q^{2 n}}+\frac{32}{5} \sum_{n=1}^{\infty} w(n) q^{n}\right\} \\
& -\left\{1+\frac{4}{5} \sum_{\substack{n=1 \\
2 \nmid n}}^{\infty} \frac{(-1)^{\frac{n-1}{2}} n^{4} q^{2 n}}{1-q^{2 n}}+\frac{64}{5} \sum_{n=1}^{\infty} \frac{n^{4} q^{2 n}}{1+q^{4 n}}+\frac{32}{5} \sum_{n=1}^{\infty} w(n) q^{2 n}\right\}
\end{aligned}
$$




$$
\begin{aligned}
& =\frac{4}{5} \sum_{\substack{n=1 \\
2 \nmid n}}^{\infty}(-1)^{(n-1) / 2} n^{4} \frac{q^{n}}{1-q^{2 n}}+\frac{64}{5} \sum_{n=1}^{\infty} n^{4} \frac{q^{n}\left(1-q^{n}\right)\left(1-q^{3 n}\right)}{\left(1+q^{2 n}\right)\left(1+q^{4 n}\right)} \\
& \quad+\frac{32}{5} \sum_{n=1}^{\infty} w^{*}(n) q^{n} .
\end{aligned}
$$

From [3, p. 256], we put $r_{12}(n)=8 \sigma_{5}(n)-512 \sigma_{5}(n / 4)+16 \beta(n)$. Hence

$$
\begin{aligned}
\varphi^{12}(q)= & +8 \sum_{n=1}^{\infty}\left\{\sigma_{5}(n)-4^{5} \sigma_{5}\left(\frac{n}{4}\right)\right\} q^{n}+\frac{15}{2} \sum_{n=1}^{\infty} 4^{5} \sigma_{5}\left(\frac{n}{4}\right) q^{n} \\
& +16 \sum_{n=1}^{\infty} \beta(n) q^{n} \\
= & +8 \sum_{n=1}^{\infty} \sum_{d \mid n} d^{5} q^{n}+\frac{15}{2} \sum_{n=1}^{\infty} \sum_{d \mid n} d^{5} q^{n}+16 \sum_{n=1}^{\infty} \beta(n) q^{n} \\
= & 1+8 \sum_{n=1}^{\infty} \frac{n^{5} q^{n}}{1-q^{n}}+\frac{15}{2} \sum_{n=1}^{\infty} \frac{n^{5} q^{n}}{1-q^{n}}+16 \sum_{4 \mid n}^{\infty} \beta(n) q^{n},
\end{aligned}
$$

where $\sum_{n=1}^{\infty} \beta(n) q^{n}:=q \prod_{n=1}^{\infty}\left(1-q^{2 n}\right)^{12}, q \in \mathbb{C},|q|<1$.

Lemma 3.3. Let $n \in \mathbb{N}$. Then we obtain

$$
\begin{aligned}
\varphi^{12, *}(q) & =\sum_{n=1}^{\infty}\left\{8 \sigma_{5}^{*}(n)-512 \sigma_{5}^{*}\left(\frac{n}{4}\right)+16 \beta^{*}(n)\right\} q^{n} \\
& =8 \sum_{\substack{n=1 \\
4 \nmid n}}^{\infty} n^{5} \frac{q^{n}}{1-q^{2 n}}+\frac{15}{2} \sum_{\substack{n=1 \\
4 \mid n}}^{\infty} n^{5} \frac{q^{n}}{1-q^{2 n}}+16 \sum_{n=1}^{\infty} \beta^{*}(n) q^{n},
\end{aligned}
$$

where

$$
\begin{aligned}
\sum_{n=1}^{\infty} \beta^{*}(n) q^{n} & :=\sum_{n=1}^{\infty}\left\{\beta(n)-\beta\left(\frac{n}{2}\right)\right\} q^{n} \\
& =q \prod_{n=1}^{\infty}\left(1-q^{2 n}\right)^{12}-q^{2} \prod_{n=1}^{\infty}\left(1-q^{4 n}\right)^{12}
\end{aligned}
$$


Proof. Since $r_{12}(n)=8 \sigma_{5}(n)-512 \sigma_{5}(n / 4)+16 \beta(n)$, we have

$$
\begin{aligned}
\varphi^{12, *}(q)= & \sum_{n=1}^{\infty}\left\{r_{12}(n)-r_{12}\left(\frac{n}{2}\right)\right\} q^{n} \\
= & \sum_{n=1}^{\infty}\left[\left\{8 \sigma_{5}(n)-512 \sigma_{5}\left(\frac{n}{4}\right)+16 \beta(n)\right\}\right. \\
& \left.\quad-\left\{8 \sigma_{5}\left(\frac{n}{2}\right)-512 \sigma_{5}\left(\frac{n}{8}\right)+16 \beta\left(\frac{n}{2}\right)\right\}\right] q^{n} \\
= & \sum_{n=1}^{\infty}\left\{8 \sigma_{5}^{*}(n)-512 \sigma_{5}^{*}\left(\frac{n}{4}\right)+16 \beta^{*}(n)\right\} q^{n} .
\end{aligned}
$$

Also, by (20), we obtain

$$
\begin{aligned}
& \varphi^{12, *}(q)=\varphi^{12}(q)-\varphi^{12}\left(q^{2}\right) \\
& =\left\{1+8 \sum_{\substack{n=1 \\
4 \nmid n}}^{\infty} \frac{n^{5} q^{n}}{1-q^{n}}+\frac{15}{2} \sum_{\substack{n=1 \\
4 \mid n}}^{\infty} \frac{n^{5} q^{n}}{1-q^{n}}+16 \sum_{n=1}^{\infty} \beta(n) q^{n}\right\} \\
& -\left\{1+8 \sum_{\substack{n=1 \\
4 \nmid n}}^{\infty} \frac{n^{5} q^{2 n}}{1-q^{2 n}}+\frac{15}{2} \sum_{\substack{n=1 \\
4 \mid n}}^{\infty} \frac{n^{5} q^{2 n}}{1-q^{2 n}}+16 \sum_{n=1}^{\infty} \beta(n) q^{2 n}\right\} \\
& =8 \sum_{\substack{n=1 \\
4 \mid n}}^{\infty} n^{5} \frac{q^{n}}{1-q^{2 n}}+\frac{15}{2} \sum_{\substack{n=1 \\
4 \mid n}}^{\infty} n^{5} \frac{q^{n}}{1-q^{2 n}}+16 \sum_{n=1}^{\infty} \beta^{*}(n) q^{n} .
\end{aligned}
$$

Remark 3.4. From (10) we can know that

$$
\varphi^{8}(-q)=16\left\{\sum_{\substack{n=1 \\ 2 \nmid n}}^{\infty} n^{3}\left(\frac{-q^{n}}{1-q^{n}}\right)+\sum_{\substack{n=1 \\ 2 \mid n}}^{\infty} \frac{n^{3} q^{n}}{1-q^{n}}\right\} .
$$

Then subtracting (10) by (21) we obtain

$$
\varphi^{8}(q)-\varphi^{8}(-q)=32 \sum_{\substack{n=1 \\ 2 \nmid n}}^{\infty} \frac{n^{3} q^{n}}{1-q^{2 n}}=32 \sum_{n=1}^{\infty} \sigma_{3}(2 n-1) q^{2 n-1} .
$$

In a similar manner, we can see that 


$$
\varphi^{12}(q)=1+8 \sum_{\substack{n=1 \\ 4 \nmid n}}^{\infty} \frac{n^{5} q^{n}}{1-q^{n}}+\frac{15}{2} \sum_{\substack{n=1 \\ 4 \mid n}}^{\infty} \frac{n^{5} q^{n}}{1-q^{n}}+16 \sum_{n=1}^{\infty} \beta(n) q^{n}
$$

from (20). Thus

$$
\varphi^{12}(-q)=1+8 \sum_{\substack{n=1 \\ 4 \nmid n}}^{\infty} \frac{n^{5}(-q)^{n}}{1-(-q)^{n}}+\frac{15}{2} \sum_{\substack{n=1 \\ 4 \mid n}}^{\infty} \frac{n^{5} q^{n}}{1-q^{n}}-16 \sum_{n=1}^{\infty} \beta(n) q^{n} .
$$

Then we have

$$
\begin{aligned}
& \varphi^{12}(q)-\varphi^{12}(-q)=8\left\{\sum_{\substack{n=1 \\
4 \nmid n}}^{\infty} \frac{n^{5} q^{n}}{1-q^{n}}-\sum_{\substack{n=1 \\
4 \nmid n}}^{\infty} \frac{n^{5}(-q)^{n}}{1-(-q)^{n}}\right\}+32 \sum_{n=1}^{\infty} \beta(n) q^{n} \\
& =8\left[\left\{\sum_{\substack{n=1 \\
4 \nmid n \\
2 \mid n}}^{\infty} \frac{n^{5} q^{n}}{1-q^{n}}+\sum_{\substack{n=1 \\
2 \nmid n}}^{\infty} \frac{n^{5} q^{n}}{1-q^{n}}\right\}-\left\{\sum_{\substack{n=1 \\
4 \nmid n \\
2 \mid n}}^{\infty} \frac{n^{5} q^{n}}{1-q^{n}}-\sum_{\substack{n=1 \\
2 \nmid n}}^{\infty} \frac{n^{5} q^{n}}{1+q^{n}}\right\}\right] \\
& \quad+32 \sum_{n=1}^{\infty} \beta(n) q^{n} \sum_{\substack{n=1 \\
2 \nmid n}}^{\infty} \frac{n^{5} q^{n}}{1-q^{2 n}}+32 \sum_{n=1}^{\infty} \beta(n) q^{n} \\
& =16 \sum_{n=1}^{\infty} \sigma_{5}(2 n-1) q^{2 n-1}+32 q \prod_{n=1}^{\infty}\left(1-q^{2 n}\right)^{12} .
\end{aligned}
$$

\section{Property of $r_{16}(n)$}

There is a simple convolution formula relating the number $r_{k}(n)$ of representations of a positive integer $n$ as the sum of $k$ squares to the number $r_{e}(n)$ of representations of $n$ as the sum of $e$ squares and $r_{k-e}(n)$. We have : 
Proposition 4.1. (See $[3$, p. 120]) Let $n, e, k \in \mathbb{N}$ satisfy $2 \leq e \leq$ $k-2$. Then we have

$$
r_{k}(n)=\sum_{l=0}^{n} r_{e}(l) r_{k-e}(n-l) .
$$

Lemma 4.2. Let $n \in \mathbb{N}$. Then we have

$$
r_{16}(n)=\frac{32}{17}\left\{\sigma_{7}(n)-2 \sigma_{7}\left(\frac{n}{2}\right)+256 \sigma_{7}\left(\frac{n}{4}\right)+16 b(n)+256 b\left(\frac{n}{2}\right)\right\},
$$

where $\sum_{n=1}^{\infty} b(n) q^{n}:=q \prod_{n=1}^{\infty}\left(1-q^{n}\right)^{8}\left(1-q^{2 n}\right)^{8}$.

Proof. Using Proposition 4.1 we can write as

$$
r_{16}(n)=\sum_{k=0}^{n} r_{8}(k) r_{8}(n-k) .
$$

Since $r_{8}(0)=1$ and $[3,(19.10)],(23)$ becomes

$$
\begin{aligned}
r_{16}(n) & -2 r_{8}(n)=\sum_{k=1}^{n-1} r_{8}(k) r_{8}(n-k) \\
=\sum_{k=1}^{n-1} & \left\{16 \sigma_{3}(k)-32 \sigma_{3}\left(\frac{k}{2}\right)+256 \sigma_{3}\left(\frac{k}{4}\right)\right\} \\
& \times\left\{16 \sigma_{3}(N-k)-32 \sigma_{3}\left(\frac{N-k}{2}\right)+256 \sigma_{3}\left(\frac{N-k}{4}\right)\right\} \\
=256 & \sum_{k=1}^{n-1} \sigma_{3}(k) \sigma_{3}(n-k)-512 \sum_{k<n / 2} \sigma_{3}(n-2 k) \sigma_{3}(k) \\
& +4096 \sum_{k<n / 4} \sigma_{3}(n-4 k) \sigma_{3}(k)-512 \sum_{k<n / 2} \sigma_{3}(k) \sigma_{3}(n-2 k) \\
& +1024 \sum_{k<n / 2} \sigma_{3}(k) \sigma_{3}\left(\frac{n}{2}-k\right)-8192 \sum_{k<n / 4} \sigma_{3}\left(\frac{n}{2}-2 k\right) \sigma_{3}(k) \\
& +4096 \sum_{k<n / 4} \sigma_{3}(k) \sigma_{3}(n-4 k)-8192 \sum_{k<n / 4} \sigma_{3}(k) \sigma_{3}\left(\frac{n}{2}-2 k\right) \\
& +65536 \sum_{k<n / 4} \sigma_{3}(k) \sigma_{3}\left(\frac{n}{4}-k\right) .
\end{aligned}
$$

Then we refer to 


$$
\sum_{k=1}^{n-1} \sigma_{3}(k) \sigma_{3}(n-k)=\frac{1}{120}\left\{\sigma_{7}(n)-\sigma_{3}(n)\right\}
$$

in $[2,(3.17)]$,

$$
\begin{gathered}
\sum_{k<n / 2} \sigma_{3}(k) \sigma_{3}(n-2 k)=\frac{1}{2040} \sigma_{7}(n)+\frac{2}{255} \sigma_{7}\left(\frac{n}{2}\right)-\frac{1}{240} \sigma_{3}(n) \\
-\frac{1}{240} \sigma_{3}\left(\frac{n}{2}\right)+\frac{1}{272} b(n)
\end{gathered}
$$

in $[1$, Theorem 5.2$]$ and

$$
\begin{aligned}
\sum_{k<n / 2} \sigma_{3}(k) \sigma_{3}(n-4 k)= & \frac{1}{32640} \sigma_{7}(n)+\frac{1}{2176} \sigma_{7}\left(\frac{n}{2}\right)+\frac{2}{255} \sigma_{7}\left(\frac{n}{4}\right) \\
& -\frac{1}{240} \sigma_{3}(n)-\frac{1}{240} \sigma_{3}\left(\frac{n}{4}\right)+\frac{9}{2176} b(n) \\
& +\frac{9}{136} b\left(\frac{n}{2}\right)
\end{aligned}
$$

in [1, Theorem 5.2].

Remark 4.3. In $[1$, p. 49] we can see that

$$
r_{16}(n)=\frac{32}{17}(-1)^{n-1}\left\{\sigma_{7}(n)-256 \sigma_{7}\left(\frac{n}{2}\right)+16 b(n)\right\},
$$

$n \in \mathbb{N}$. When $n$ is an even integer, equating (24) with Theorem 4.2, we have

$$
128 b\left(\frac{b}{2}\right)+16 b(n)=-\sigma_{7}(n)+129 \sigma_{7}\left(\frac{n}{2}\right)-128 \sigma_{7}\left(\frac{n}{4}\right)=-\sigma_{7, o o}(n),
$$

where $\sigma_{7, o o}(n):=\sum_{\substack{d \mid n \\ d \\ \frac{n}{d} \text { odd } \\ \text { odd }}} d^{7}$. Note that $\sigma_{s, o o}(n)=\sigma_{s}(n)-\left(2^{s}+1\right) \sigma_{s}\left(\frac{n}{2}\right)+$ $2^{s} \sigma_{s}\left(\frac{n}{4}\right)$ in $\left[3\right.$, p. 35]. And we can deduce that if $n$ is even then $\sigma_{7, o o}(n)=$ 0 . Therefore,

$$
b(n)=-8 b\left(\frac{n}{2}\right) .
$$

Lemma 4.4. Let $n \in \mathbb{N}$. Then we obtain

$$
\begin{aligned}
\varphi^{16, *}(q)= & \frac{32}{17} \sum_{n=1}^{\infty}\left\{\sigma_{7}^{*}(n)-2 \sigma_{7}^{*}\left(\frac{n}{2}\right)+256 \sigma_{7}^{*}\left(\frac{n}{4}\right)+16 b^{*}(n)\right. \\
& \left.+256 b^{*}\left(\frac{n}{2}\right)\right\} q^{n},
\end{aligned}
$$


where

$$
\begin{aligned}
\sum_{n=1}^{\infty} b^{*}(n) q^{n} & :=\sum_{n=1}^{\infty}\left\{b(n)-b\left(\frac{n}{2}\right)\right\} q^{n} \\
& =q \prod_{n=1}^{\infty}\left(1-q^{n}\right)^{8}\left(1-q^{2 n}\right)^{8}-q^{2} \prod_{n=1}^{\infty}\left(1-q^{2 n}\right)^{8}\left(1-q^{4 n}\right)^{8} .
\end{aligned}
$$

Proof. By Lemma 4.2 we have

$$
\begin{aligned}
& \varphi^{16, *}(q)=\sum_{n=1}^{\infty}\left\{r_{16}(n)-r_{16}\left(\frac{n}{2}\right)\right\} q^{n} \\
& =\sum_{n=1}^{\infty}\left[\frac{32}{17}\left\{\sigma_{7}(n)-2 \sigma_{7}\left(\frac{n}{2}\right)+256 \sigma_{7}\left(\frac{n}{4}\right)+16 b(n)+256 b\left(\frac{n}{2}\right)\right\}\right. \\
& \left.\quad \quad-\frac{32}{17}\left\{\sigma_{7}\left(\frac{n}{2}\right)-2 \sigma_{7}\left(\frac{n}{4}\right)+256 \sigma_{7}\left(\frac{n}{8}\right)+16 b\left(\frac{n}{2}\right)+256 b\left(\frac{n}{4}\right)\right\}\right] q^{n} \\
& =\frac{32}{17} \sum_{n=1}^{\infty}\left\{\sigma_{7}^{*}(n)-2 \sigma_{7}^{*}\left(\frac{n}{2}\right)+256 \sigma_{7}^{*}\left(\frac{n}{4}\right)+16 b^{*}(n)+256 b^{*}\left(\frac{n}{2}\right)\right\} q^{n},
\end{aligned}
$$

where

$$
\begin{aligned}
& \sum_{n=1}^{\infty} b^{*}(n) q^{n}:=\sum_{n=1}^{\infty}\left\{b(n)-b\left(\frac{n}{2}\right)\right\} q^{n}=\sum_{n=1}^{\infty}\left\{b(n) q^{n}-b(n) q^{2 n}\right\} \\
& =q \prod_{n=1}^{\infty}\left(1-q^{n}\right)^{8}\left(1-q^{2 n}\right)^{8}-q^{2} \prod_{n=1}^{\infty}\left(1-q^{2 n}\right)^{8}\left(1-q^{4 n}\right)^{8} .
\end{aligned}
$$

Thus the proof is complete.

Example 4.5. We list first tenth values of $r_{16}^{*}(n)$.

\begin{tabular}{|c|c|c|c|c|c|c|c|c|c|c|}
\hline$n$ & 1 & 2 & 3 & 4 & 5 & 6 & 7 & 8 & 9 & 10 \\
\hline$r_{16}^{*}(n)$ & 32 & 448 & 4480 & 28672 & 140736 & 521472 & 1580800 & 3964928 & 8945824 & 18485376 \\
\hline
\end{tabular}

$$
\text { TABLE 1. Some values of } r_{16}^{*}(n)
$$

Lemma 4.6. Let $n$ be an odd integer. Then we have $\sigma_{7}(n) \equiv b(n)$ $(\bmod 17)$.

Proof. Using Lemma 4.2 for odd $n$, we obtain

$$
r_{16}(n)=\frac{32}{17}\left\{\sigma_{7}(n)+16 b(n)\right\} .
$$

So $\sigma_{7}(n)+16 b(n) \equiv 0(\bmod 17)$. It follows that

$$
\sigma_{7}(n) \equiv-16 b(n) \equiv b(n) \quad(\bmod 17) .
$$


Theorem 4.7. Let $n \in \mathbb{N}$. Then we have

(a) $r_{16}(n) \equiv 32 \sigma_{7}(n)(\bmod 64)$.

(b) $r_{16}(n) \equiv 0(\bmod 32)$.

(c) Let $n=p_{1}^{e_{1}} p_{2}^{e_{2}} \cdots p_{r}^{e_{r}}$. If $p_{i}$ and $e_{i}$ are odd, then $r_{16}(n) \equiv 0$ $(\bmod 64)$.

(d)

$r_{16}(n) \equiv \begin{cases}32 \quad(\bmod 64), & \text { if } n=2^{m}(m \geq 0) \text { or } t^{2} \text { or } 2^{m} t^{2}, \\ 0 \quad(\bmod 64), & \text { otherwise. }\end{cases}$

Proof. (a) By Lemma 4.2, we obtain

$$
\begin{aligned}
17 r_{16}(n)= & 32 \sigma_{7}(n)-64 \sigma_{7}\left(\frac{n}{2}\right)+32 \cdot 256 \sigma_{7}\left(\frac{n}{4}\right)+32 \cdot 16 b(n) \\
& +32 \cdot 256 b\left(\frac{n}{2}\right) .
\end{aligned}
$$

So we have

$$
17 r_{16}(n) \equiv 32 \sigma_{7}(n) \quad(\bmod 64) .
$$

Since $(49,64)=1,(25)$ becomes

$49 \cdot 17 r_{16}(n) \equiv 49 \cdot 32 \sigma_{7}(n) \equiv(2 \cdot 24+1) 32 \sigma_{7}(n) \equiv 32 \sigma_{7}(n) \quad(\bmod 64)$.

Here $49 \cdot 17 \equiv 1(\bmod 64)$, so we deduce that $r_{16}(n) \equiv 32 \sigma_{7}(n)$ $(\bmod 64)$.

(b) By (25), we have $17 r_{16}(n)=32 \sigma_{7}(n)+64 k$ for some integer $k$.

Thus $17 r_{16}(n) \equiv 0(\bmod 32)$. Since $(17,32)=1$, we have $r_{16}(n) \equiv$ $0(\bmod 32)$.

(c) Let $n=p_{1}^{e_{1}} p_{2}^{e_{2}} \cdots p_{r}^{e_{r}}$ and $p_{i}=p_{1}$, then we can see that

$$
\begin{aligned}
\sigma_{7}(n) & =\sigma_{7}\left(p_{1}^{e_{1}} p_{2}^{e_{2}} \cdots p_{r}^{e_{r}}\right)=\sigma_{7}\left(p_{1}^{e_{1}}\right) \sigma_{7}\left(\frac{n}{p_{1}^{e_{1}}}\right) \\
& =\left\{1+p_{1}^{7}+p_{1}^{14}+\cdots+p_{1}^{7 e_{1}}\right\} \sigma_{7}\left(\frac{n}{p_{1}^{e_{1}}}\right) \\
& \equiv 0 \quad(\bmod 2) .
\end{aligned}
$$

Therefore, by Theorem 4.7 (a), we obtain

$$
17 r_{16}(n) \equiv 32 \sigma_{7}(n) \equiv 0 \quad(\bmod 64) .
$$

Since $(17,64)=1$, so $r_{16}(n) \equiv 0(\bmod 64)$.

(d) The proof is similar to Theorem 4.7 (c). If $n=2^{m}$, then

$$
\sigma_{7}(n)=\sigma_{7}\left(2^{m}\right)=1+2^{7}+2^{14}+\cdots+2^{7 m} \equiv 1 \quad(\bmod 2) .
$$


It means that $\sigma_{7}(n)=2 k+1$ for some integer $k$. Thus, by Theorem 4.7 (a), we obtain

$$
17 r_{16}(n) \equiv 32 \sigma_{7}(n) \equiv 32(2 k+1) \equiv 32 \quad(\bmod 64) .
$$

Since $(17,64)=1$, so $r_{16}(n) \equiv 32(\bmod 64)$. The other cases are similar.

Corollary 4.8. We have $r_{16}\left(2^{s} t^{2 l} m\right) \equiv r_{16}(m)(\bmod 64)$.

Proof. We deduce from Theorem 4.7 (d).

Theorem 4.9. Let $n$ be an odd integer. Then we have $r_{16}(n) \equiv$ $32 \sigma_{7}(n)(\bmod 512)$.

Proof. In Lemma 4.4, if $n$ is odd then

$$
\varphi^{16, *}(q)=\sum_{r=1}^{\infty} r_{16}(n) q^{n}=\frac{32}{17} \sum_{n=1}^{\infty}\left\{\sigma_{7}(n)+16 b(n)\right\} q^{n} .
$$

So

$$
\sum_{r=1}^{\infty} 17 r_{16}(n) q^{n}=\sum_{n=1}^{\infty}\left\{32 \sigma_{7}(n)+32 \cdot 16 b(n)\right\} q^{n} .
$$

Therefore

$$
17 r_{16}(n) \equiv 32 \sigma_{7}(n) \quad(\bmod 512) .
$$

Since $(17,512)=1,(26)$ is

$$
\begin{aligned}
241 \cdot 17 r_{16}(n) & \equiv 241 \cdot 32 \sigma_{7}(n) \equiv(512 \cdot 15+32) \sigma_{7}(n) \\
& \equiv 32 \sigma_{7}(n) \quad(\bmod 512) .
\end{aligned}
$$

Because $241 \cdot 17 \equiv 1(\bmod 512)$, so we deduce that $r_{16}(n) \equiv 32 \sigma_{7}(n)$ $(\bmod 512)$.

\section{Appendix}

The first forty five values of $b(n)$ are given in Table 2 . 


\begin{tabular}{|c|c||c|c||c|c|}
\hline$n$ & $b(n)$ & $n$ & $b(n)$ & $n$ & $b(n)$ \\
\hline 1 & 1 & 16 & 4096 & 31 & 227552 \\
\hline 2 & -8 & 17 & 14706 & 32 & -32768 \\
\hline 3 & 12 & 18 & 16344 & 33 & 13104 \\
\hline 4 & 64 & 19 & -39940 & 34 & -117648 \\
\hline 5 & -210 & 20 & -13440 & 35 & -213360 \\
\hline 6 & -96 & 21 & 12192 & 36 & -130752 \\
\hline 7 & 1016 & 22 & -8736 & 37 & 160526 \\
\hline 8 & -512 & 23 & 68712 & 38 & 319520 \\
\hline 9 & -2043 & 24 & -6144 & 39 & 16584 \\
\hline 10 & 1680 & 25 & -34025 & 40 & 107520 \\
\hline 11 & 1092 & 26 & -11056 & 41 & 10842 \\
\hline 12 & 768 & 27 & -50760 & 42 & -97536 \\
\hline 13 & 1382 & 28 & 65024 & 43 & -630748 \\
\hline 14 & -8128 & 29 & -102570 & 44 & 69888 \\
\hline 15 & -2520 & 30 & 20160 & 45 & 429030 \\
\hline
\end{tabular}

TABLE $2 . b(n)$ for $n(1 \leq n \leq 45)$

\section{References}

[1] N. Cheng and K. S. Williams, Evaluation of some convolution sums involving the sum of divisors functions, Yokohama Mathematical J., 52 (2005), 39-57.

[2] J. G. Huard, Z. M. Ou, B. K. Spearman, and K. S. Williams, Elementary Evaluation of Certain Convolution Sums Involving Divisor Functions, Number theory for the millennium, II, (2002), 229-274.

[3] K. S. Williams, Number Theory in the Spirit of Liouville, London Mathematical Society, Student Texts 76, Cambridge, (2011).

Aeran Kim

Department of Mathematics and Institute of Pure and Applied Mathematics,

Chonbuk National University,

Chonju, Chonbuk 561-756, Korea.

E-mail: ae_ran_kim@hotmail.com

Daeyeoul Kim

National Institute for Mathematical Sciences,

Yuseong-daero 1689-gil, Yuseong-Gu, Daejeon 305-811, South Korea.

E-mail: daeyeoul@nims.re.kr 
Nazli Yildiz İkikardes

Department of Elementary Mathematics Education,

Necatibey Faculty of Education, Balikesir University, 10100 Balikesir, Turkey.

E-mail: nyildizikikardes@gmail.com 